Modern Physics Letters B

(C) World Scientific Publishing Company

\title{
ERRATA
}

\section{ORBITAL MAGNETISM OF MESOSCOPIC METALS: BEYOND THE PERTURBATION THEORY}

\author{
R. A. SEROTA \\ [Mod. Phys. Lett. B, Vol. 6, No. 23 (1992) 1455-1463]
}

1. The paragraph below Eq. (8) should read

In this equation we maintained the units of the moment used in I (aside from the factor $2 / s$ omitted there) and explicitly exposed $\Delta=1$. Numerical summation in Eq. (8) leads to the magnetic field dependence of the magnetic moment such as depicted in Fig. 2 for $E_{c} / \Delta=10$ for $\varsigma=1, \varsigma=.5, \varsigma=.25$ and $\varsigma=.125$ respectively. Saturation to the asymptotic dependence clearly takes place as the temperature becomes comparable to the interlevel spacing $\Delta$.

2. In the last line of page 1461, "Ref. 22" should read "Refs. 21 and 22 ".

3. In the last line of Acknowledgements and in Ref. 16, "Eq. (5)" should read "Eq. (6)". 